Check for updates

Cite this: RSC Adv., 2019, 9, 36578

Received 19th August 2019

Accepted 29th October 2019

DOI: $10.1039 / \mathrm{c} 9 \mathrm{ra06481h}$

rsc.li/rsc-advances

\section{Ultrasensitive detection of uric acid in serum of patients with gout by a new assay based on Pt@Ag nanoflowers}

\begin{abstract}
Xue Wang, ${ }^{a}$ Shujun Chen, ${ }^{a}$ Xiaomin Tang, ${ }^{b}$ Daiqin $\operatorname{Lin}^{c}$ and Ping Qiu (D) *a
A ultrasensitive assay for the determination of uric acid (UA) based on Pt@Ag nanoflowers (Pt@Ag NFs) was constructed. $\mathrm{H}_{2} \mathrm{O}_{2}$ was formed by the reaction of uricase and UA and produced the hydroxyl radical $\left({ }^{\circ} \mathrm{OH}\right)$. The system was catalyzed by PtaAg NFs to change the color of 3,3',5,5'-tetramethylbenzidine (TMB) from colorless to blue, and the morphology and chemical properties of Pt@Ag NFs were characterized by transmission electron microscopy and X-ray photoelectron spectroscopy. Under the optimized conditions, a linear relationship between the absorbance and UA concentration was in the range of 0.5$150 \mu \mathrm{M}\left(R^{2}=0.995\right)$ with a limit of detection of $0.3 \mu \mathrm{M}(\mathrm{S} / \mathrm{N}=3)$. The method can be applied to detection of UA in actual samples with satisfactory results. The proposed assay was successfully applied to the detection of UA in human serum with recoveries over $96.8 \%$. Thus, these results imply that the UA assay provides an effective tool in fast clinical analysis of gout.
\end{abstract}

\section{Introduction}

Uric acid (UA) is the final product of human sputum metabolism, ${ }^{1}$ its concentration in serum is $120-480 \mu \mathrm{M} ;{ }^{2}$ many diseases of the human body are related to abnormal uric acid content, such as gout, Lesch-Nyhan syndrome, diabetes, hyperuricemia, heart disease..$^{3-6}$ Monitoring the concentration of UA in human serum is essential and can be used as an early warning for these diseases. Many diseases are caused by diet and lifestyle, so as a remedy, all asymptomatic gout patients should be encouraged to change their lifestyle. This requires the development of a suitable cost-effective monitoring system to provide adequate feedback and treatment guidelines for gout patients.

Gout is an inflammatory reaction that occurs primarily in the joints and forms UA crystals that cause pain. ${ }^{7}$ There is a direct positive correlation between UA levels and the future risk of gout. Specifically, as the concentration of UA increases, the risk of crystal formation increases, thereby increasing the patient's susceptibility to gout. ${ }^{8}$

There are many approaches to detect uric acid, such as, capillary electrophoresis, ${ }^{9}$ fluorometry, ${ }^{10,11}$ chromatography, ${ }^{12}$ electrochemical methods, ${ }^{13,14}$ chemiluminescence ${ }^{15}$ and colorimetry.$^{16}$ Furthermore, the colorimetric method is widely used because of its simple operation, fast analysis speed, high sensitivity in these methods. Currently, there are two main

${ }^{a}$ Department of Chemistry, Nanchang University, Nanchang, 330031, China. E-mail: pingqiu@ncu.edu.cn

${ }^{b}$ The Fourth Affiliated Hospital of Nanchang University, Nanchang, 330003, China 'Jiangxi Province Product Quality Supervision Testing Institute, Nanchang, 330047, China colorimetric methods for the detection of UA. One is a colorimetric assay based on precious metal (e.g., $\mathrm{Au}$ and $\mathrm{Ag}$ ) nanomaterials, in which uric acid can induce nanoparticle aggregation, ${ }^{17}$ etching and anti-etching ${ }^{18,19}$ to cause color changes. However, the dispersion of the nanomaterial is unstable and precipitates easily, which causes the color of the solution to change or even disappear. Many external factors also may cause undesirable changes in the state of nanomaterials, ${ }^{20}$ resulting in unsatisfactory results. The other one is the catalytic oxidation strategy, which bases on the peroxidase-like properties of nanomaterials, such as porous metal-organic framework MIL-53 (Fe), ${ }^{21}$ graphite carbonitride nanosheets ${ }^{22}$ and cobalt selenide nanosheets. ${ }^{23}$ They can change color by $\mathrm{H}_{2} \mathrm{O}_{2}$ catalytic peroxidase substrate. The peroxidase mimic enzymes have the advantages of low cost, good stability, simple preparation, controllable structure and composition, and adjustable catalytic activity. Therefore, this kind of nanomaterials have become an ideal and important colorimetric detection tool.

Precious metal nanomaterials have a wide range of applications in catalysis and assays due to their unique physical and chemical properties. ${ }^{24,25}$ More importantly, the bimetallic nanostructures have synergistic and controllable catalytic properties compared with their mono-metallic nanostructures. ${ }^{26}$ So far, nanomaterials of various shapes have been synthesized by chemical methods, such as nanowires, ${ }^{27}$ nanotubes, ${ }^{28}$ nanorods ${ }^{29}$ and nanowires. ${ }^{30}$ Platinum nanomaterials have attracted much attention due to their excellent catalytic ability and high utilization rate, and have been applied to many fields such as catalysis, battery, sensing and medical treatment. ${ }^{31-34}$ Silver nanoflowers have large specific surface area and good electrical conductivity, especially peroxidase-like 
properties, which provides an excellent substrate for the construction of the assay. ${ }^{35-37}$ A new type of Pt@Ag nanoflower (Pt@Ag NFs) was prepared by adhesion of platinum nanoparticles with high catalytic activity on the surface of silver nanoflowers to further improve the ability of reducing $\mathrm{H}_{2} \mathrm{O}_{2}$.

This paper presents a method for preparing Pt@Ag NFs using bovine serum albumin as raw material. A colorimetric assay using the peroxidase-like activity based on Pt@Ag NFs was developed to detect UA with sensitivity and high selectivity. $\mathrm{Pt} @ A g$ NFs catalyze the colorimetric system of TMB and $\mathrm{H}_{2} \mathrm{O}_{2}$ (which is produced by uricase-specific catalysis of UA), resulting in a solution color changing from colorless to blue. The color change of the solution depends indirectly on the amount of uric acid and can be judged by an ultraviolet-visible spectrophotometer and the naked eye.

\section{Experimental}

\section{Chemical medicines and instrumentations}

Silver nitrate $\left(\mathrm{AgNO}_{3}\right)$, chloroplatinic acid $\left(\mathrm{H}_{2} \mathrm{PtCl}_{6} \cdot 6 \mathrm{H}_{2} \mathrm{O}\right)$, bovine serum albumin (BSA), ascorbic acid (AA), 3,3',5, $5^{\prime}$-tetramethylbenzidine (TMB), cysteine (Cys) and glucose (Glu) were purchased in Sinopharm Chemical Reagent Co., Ltd. (Shanghai, China).

Uric acid (UA), uricase, dopamine (DA), and glutathione (GSH) were obtained from Sigma-Aldrich (Shanghai, China). Urea, hydrogen peroxide (30 wt $\left.\% \mathrm{H}_{2} \mathrm{O}_{2}\right), \mathrm{KNO}_{3}, \mathrm{Mg}\left(\mathrm{NO}_{3}\right)_{2}$, $\mathrm{NaCl}, \mathrm{CaCl}_{2}$ were obtained from Beijing Chemical Industry Co., Ltd. (Beijing, China). All reagents are analytical grade and do not require further treatment. Experimental water was ultrapure water (18.25 $\mathrm{M} \Omega$, Millipore, USA).

UV-vis spectral measurements were recorded on Agilent 8453 UV-vis spectrophotometer (Agilent Technologies, Santa Clara, CA, USA). The morphology of the Pt@Ag NFs were characterized by transmission electron microscopy (TEM, JEM-2100, JEOL Co., Japan). X-ray photoelectron spectroscopy (XPS) was carried out on EscaLab 250Xi with $\mathrm{Al} \mathrm{K} \alpha \mathrm{X}$-ray radiation as the source for excitation. Biochemical analysis was measured using biochemical analyzer (AU2007, Beckman, Olympus, USA).

\section{Preparation of Pt@Ag NFs}

The innovative synthesis of Pt@Ag NFs is the key step to this experiment. ${ }^{37}$ Briefly, BSA (5 mg mL ${ }^{-1}, 20 \mathrm{~mL}$ ) and $\mathrm{AgNO}_{3}$ $(10 \mathrm{mM}, 10 \mathrm{~mL})$ aqueous solution were mixed and stirred at room temperature for $10 \mathrm{~min}$, then $\mathrm{AA}\left(50 \mathrm{mg} \mathrm{mL} \mathrm{mL}^{-1}, 1 \mathrm{~mL}\right)$ was added into the above solution drop by drop. The color of the solution turned light grey, indicating that Ag NFs had been successfully formed. $\mathrm{H}_{2} \mathrm{PtCl}_{6} \cdot 6 \mathrm{H}_{2} \mathrm{O}(5.6 \mathrm{mM}, 10 \mathrm{~mL})$ was then mixed with the Ag NFs solution and the mixture was stirred at room temperature for a further $10 \mathrm{~min}$ until the color turned dark grey. This indicates that Pt@Ag NFs was successfully synthesized, and the obtained product was repeatedly washed three times with water and ethanol, respectively. Finally, the precipitate was re-dissolved in $20 \mathrm{~mL}$ of ultrapure water for later use.

\section{Colorimetric measurement}

$150 \mu \mathrm{L}$ of Britton-Robinson buffer (B-R buffer, $\mathrm{pH}$ 8.5) was mixed with $50 \mu \mathrm{L}, 100 \mu \mathrm{g} \mathrm{mL}^{-1}$ uricase and $50 \mu \mathrm{L}$ different concentrations of UA in a $35^{\circ} \mathrm{C}$ water bath for $15 \mathrm{~min}$. Then 200 $\mu \mathrm{L}, 16$ mM TMB, $30 \mu \mathrm{L}$ Pt@Ag NFs and $1520 \mu \mathrm{L}, 50 \mathrm{mM}$ HAcNaAc buffer of pH 4.0 were added to the above solution. Shake well to bring the mixed solution into full contact, and incubate for another $40 \mathrm{~min}$ in a $55{ }^{\circ} \mathrm{C}$ water bath, and collect the data using an UV-vis spectrometer.

\section{Analysis of UA in human serum}

The serum samples of gout patients were collected from the Nanchang University Fourth Affiliated Hospital (Nanchang city, China). The samples treatment was relatively simple. The samples were treated with $4000 \mathrm{rpm}$ centrifugation for $15 \mathrm{~min}$, and the supernatant diluted with phosphate buffer saline (PBS, pH 7.4). The analysis of each serum sample was repeated three times.

\section{Results and discussion}

\section{Principle of colorimetric assay}

Scheme 1 simply describes the mechanism of the detection of UA by Pt@Ag NFs, which also involves the synthesis of Pt@Ag NFs and the oxidation of uricase. Under weak alkaline conditions, uricase specifically catalyzes UA, producing allantoin, carbon dioxide and $\mathrm{H}_{2} \mathrm{O}_{2}$. Pt@Ag NFs have peroxidase-like activity, which further catalyzes the decomposition of $\mathrm{H}_{2} \mathrm{O}_{2}$ to form hydroxyl radicals $\left({ }^{\circ} \mathrm{OH}\right)$. ${ }^{\circ} \mathrm{OH}$ oxidizes the peroxidase substrate TMB (colorless) to oxidized TMB (oxTMB, blue). These two steps can be expressed by eqn (1) and (2):

$$
\begin{gathered}
\text { Uric acid }+\mathrm{O}_{2}+\mathrm{H}_{2} \mathrm{O} \underset{\mathrm{pH} 8.5}{\stackrel{\text { uricase }}{\longrightarrow}} \text { allantoin }+\mathrm{H}_{2} \mathrm{O}_{2}+\mathrm{CO}_{2} \\
\mathrm{H}_{2} \mathrm{O}_{2}+\mathrm{TMB} \underset{\mathrm{pH} 4}{\stackrel{\mathrm{Pt} @ \mathrm{Ag} \mathrm{NFs}}{\longrightarrow}} \mathrm{H}_{2} \mathrm{O}+\text { oxidized TMB }
\end{gathered}
$$

In order to verify the principle of the colorimetric assay, the UV-vis spectra of TMB and the mixture of UA, uricase, Pt@Ag NFs and $\mathrm{H}_{2} \mathrm{O}_{2}$ were compared. As shown in Fig. 1, when TMB

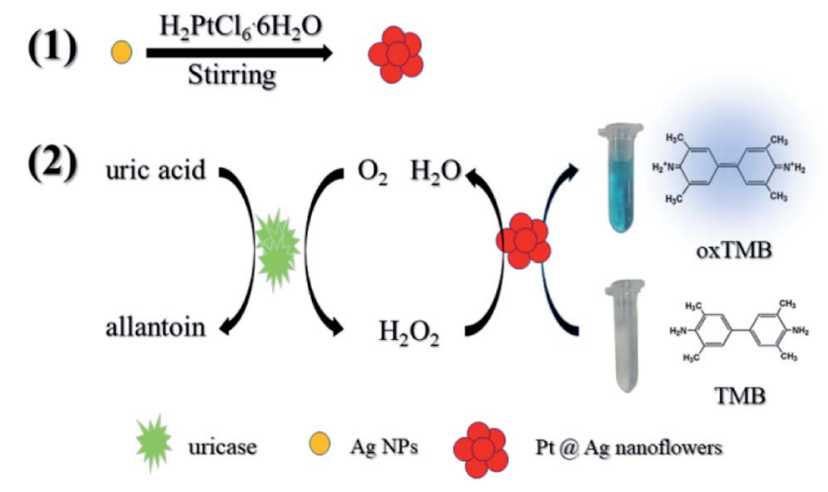

Scheme 1 Schematic diagram of the colorimetric detection of UA using $\mathrm{H}_{2} \mathrm{O}_{2}$ and Pt@Ag NFs catalytic oxidation of TMB. 


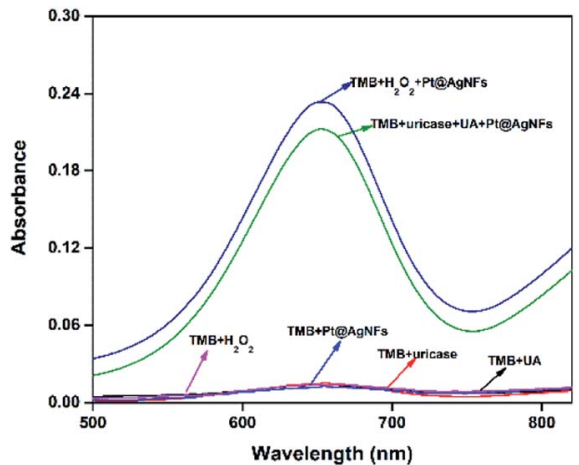

Fig. 1 UV-vis spectra of TMB interacting with different substrates. (1) $\mathrm{TMB}+\mathrm{UA}$; (2) TMB + uricase; (3) TMB + Pt@Ag NFs; (4) TMB + $\mathrm{H}_{2} \mathrm{O}_{2}$; (5) $\mathrm{TMB}+\mathrm{UA}+$ uricase + Pt@Ag NFs; (6) $\mathrm{TMB}+\mathrm{H}_{2} \mathrm{O}_{2}+$ Pt@Ag NFs; conditions: UA, $20 \mu \mathrm{M}$; uricase, $1.25 \mu \mathrm{g} \mathrm{mL;}$ Pt@Ag NFs, $30 \mu \mathrm{L} ; \mathrm{TMB}$, $1.6 \mathrm{mM} ; \mathrm{H}_{2} \mathrm{O}_{2}, 20 \mu \mathrm{M}$.

was only mixed with one of the above substances, there was no significant absorption peak of oxTMB at $652 \mathrm{~nm}$ (black, red, blue and purple curves). In addition, when TMB was added to the mixed solution of Pt@Ag NFs, UA and uricase, the absorbance increased obviously at $652 \mathrm{~nm}$ (green curve). At the same time, when $\mathrm{H}_{2} \mathrm{O}_{2}$ was mixed with TMB and Pt@Ag NFs (dark blue curve), a similar phenomenon can be observed, and the absorbance increases. This indicates that Pt@Ag NFs catalyzes $\mathrm{H}_{2} \mathrm{O}_{2}$ and TMB in the same way as UA, uricase and TMB. The results of the whole experiment demonstrated that UA was first catalyzed by uricase to produce $\mathrm{H}_{2} \mathrm{O}_{2}$, and then further catalyzed $\mathrm{H}_{2} \mathrm{O}_{2}$ by Pt@Ag NFs, and the color of reaction substrate TMB changed. Therefore, it can be seen that there is a significant color change from colorless to blue in Scheme 1.

\section{Structure and properties of Pt@Ag NFs nanoflower}

Pt@Ag NFs play a crucial role in the detection of colorimetric uric acid assays. The structure and morphology of Pt@Ag NFs were studied by transmission electron microscopy. Fig. 2a shows the overall morphology of Pt@Ag NFs. The product consists of a large number of flower-like structures with an average diameter of $50 \mathrm{~nm}$, which are well dispersed in water. The magnified TEM image in Fig. 2b shows that the flower-like Pt@Ag nanoparticles are surrounded by a thin layer of BSA. Energy dispersive spectroscopy (EDS) is a method for characterizing elemental composition and X-ray photoelectron spectroscopy (XPS) was performed to confirm the elemental composition and oxidation state of the prepared nanocomposite. ${ }^{38-40}$ Fig. 2c shows the EDS spectrum of Pt@Ag NFs, confirming the presence of Pt and Ag in Pt@Ag NFs. The oxidation states and formations of Pt@Ag NFs were determined using XPS measurements. A survey XPS spectrum confirm the coexistence of Pt and Ag elements in Pt@Ag NFs (Fig. 2d). The oxidation states of $\mathrm{Pt}$ and $\mathrm{Ag}$ are obtained by fitting the peaks in high resolution Pt $4 \mathrm{f}$ and $\mathrm{Ag} 3 \mathrm{~d}$ XPS spectra (Fig. 2e and f), where the peaks at 70.0, 73.2,366.9, and $372.9 \mathrm{eV}$ correspond to Pt $4 f_{7 / 2}$, Pt $4 f_{5 / 2}, A g 3 d_{5 / 2}$, and $A g 3 d_{3 / 2}$, respectively, verifying the formation of $\mathrm{Pt}$ and $\mathrm{Ag}$.

\section{Optimization of sensing system}

It is known from previous literature that the optimal $\mathrm{pH}$, incubation temperature and incubation time for uricasecatalyzed UA reaction are $8.5,35{ }^{\circ} \mathrm{C}$ and $15 \mathrm{~min}$, respectively. Relative activity was used to judge the optimal value of reaction parameters, which can be represented by $\left(A-A_{0}\right) / A$ ( $A$ is the absorbance in the presence of UA, $A_{0}$ is in the absence of UA).
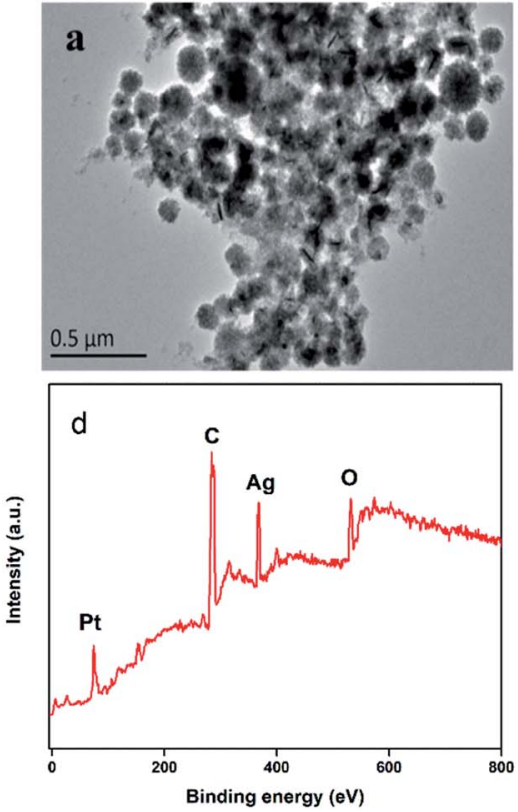
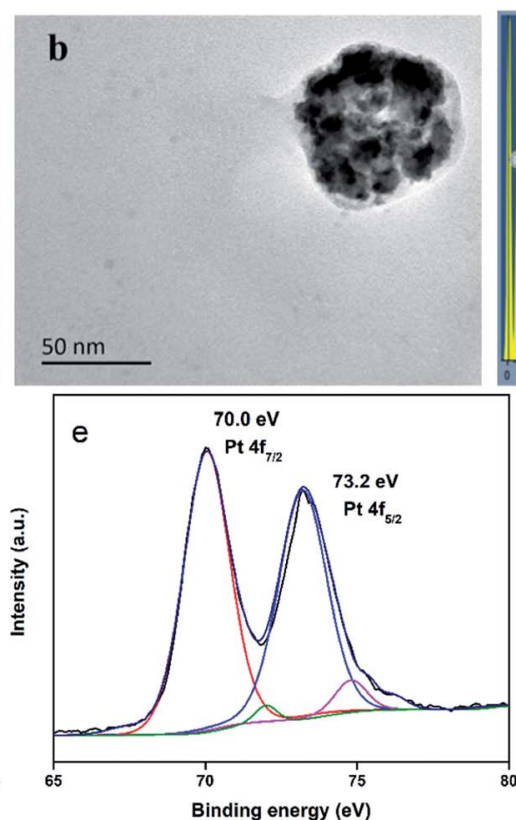
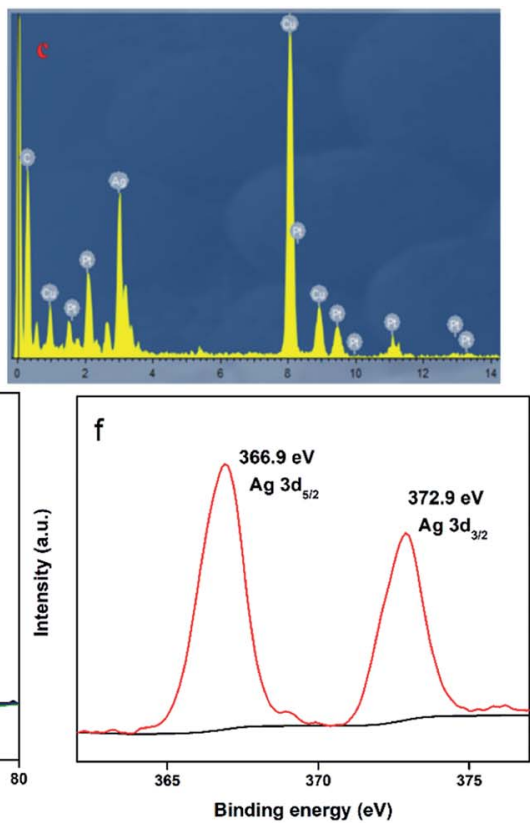

Fig. 2 Characterization of the Pt@Ag NFs: (a) TEM image; (b) magnified images; (c) energy dispersive spectra; (d) the XPS spectrum of the Pt@Ag NFs, (e) the high resolution Pt 4f; (f) the high resolution Ag 3d, respectively. 

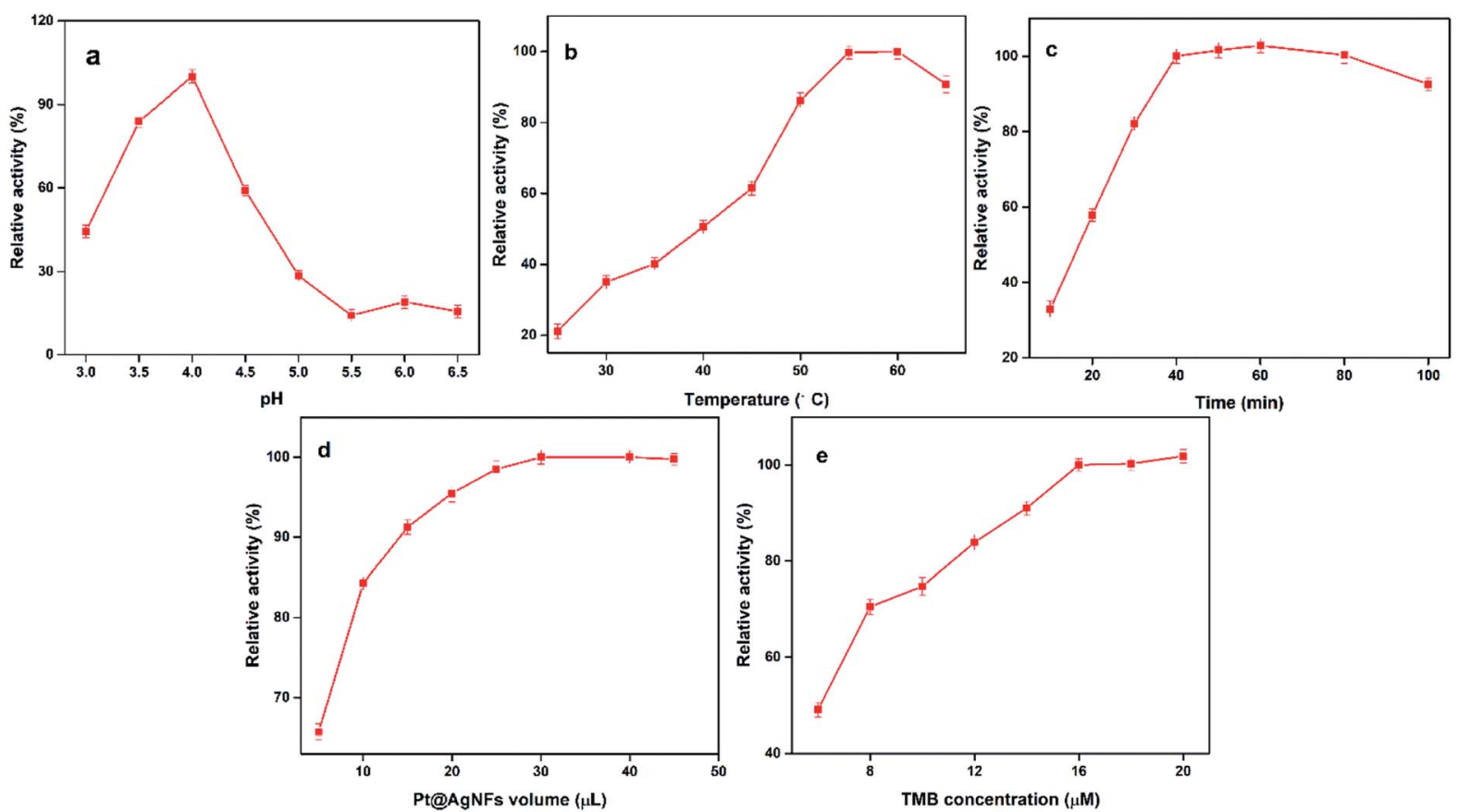

Fig. 3 Effects the peroxidase - like activity of Pt@Ag NFs (a) pH, (b) incubation temperature, (c) incubation time (d) Pt@Ag NFs volume and (e) TMB concentration.

Next, the catalytic activity of Pt@Ag NFs catalyst was optimized to obtain the best sensing response of UA assay. These parameters include $\mathrm{pH}$, incubation temperature, incubation time, Pt@Ag NFs volume and TMB concentration. As can be seen from Fig. 3a, with the increase of $\mathrm{pH}$ (3.0-6.5), the relative activity at $652 \mathrm{~nm}$ increases firstly and then decreases. At $\mathrm{pH}=$ 4.0 , the relative activity is the maximum. The catalytic activity of nanozymes at $25-65^{\circ} \mathrm{C}$ was studied (Fig. 3b). With the increase of temperature, the intensity of absorption peak at $652 \mathrm{~nm}$ gradually increased to the stage of platform, and the incubation temperature was easy to control, which was convenient for practical operation. Fig. 3c shows the effect of incubation time (10-100 $\mathrm{min}$ ) on the catalytic activity of Pt@Ag NFs. It can be seen from the figure that when the time is $40 \mathrm{~min}$, the relative activity tends to be maximum and there is a small fluctuation. We choose $40 \mathrm{~min}$ as the optimum time. At the same time, the volume of Pt@Ag NFs and the concentration of TMB also have a significant effect on the response of the assay. A series of Pt@Ag NFs volumes for UA detection were optimized (Fig. 3d). The relative activity increased gradually at the volume of 10-30
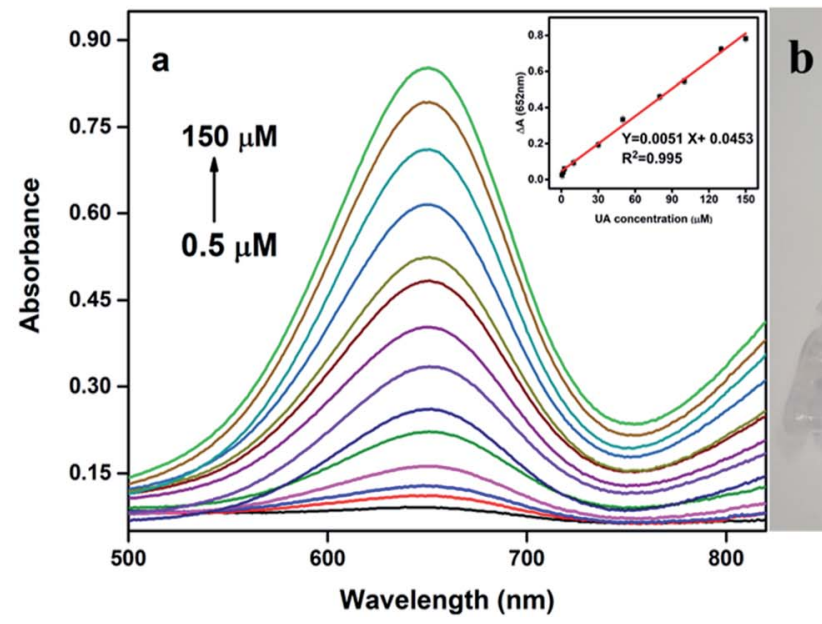

0-150 $\mu \mathrm{M}$

Fig. 4 (a) Pt@Ag NFs determination of UA (from low to high: 0.5, 1.5, 2.5, 10, 20, 30, 40, 50, 70, 80, 100, 120, 130, 150 $\mu$ M), illustrations: linear standard curve of UA; (b) a digital image of reaction systems with different amounts of UA (from left to right: $0,1.5,2.5,10,20,30,40,50,70,80$, $100,120,130,150 \mu \mathrm{M})$. 
Table 1 Comparison of analytical performances of different UA assays

\begin{tabular}{|c|c|c|c|c|c|}
\hline Assay system & Signal output & Linear range $(\mu \mathrm{M})$ & $\operatorname{LOD}(\mu \mathrm{M})$ & Real sample & Ref. \\
\hline TCPO- $\mathrm{H}_{2} \mathrm{O}_{2}$-rubrene & Chemiluminescence & $10-1000$ & 5.0 & Serum & 41 \\
\hline $\mathrm{CNCo}$ & Current & $2-110$ & 0.83 & Serum & 42 \\
\hline $\mathrm{TMB} / \mathrm{g}-\mathrm{C}_{3} \mathrm{~N}_{4} /$ uricase & Colorimetry & $10-100$ & 8.9 & NR & 22 \\
\hline $\mathrm{Ag}$ nanoprism/uricase & Colorimetry & $1-40$ & 0.7 & Serum & 16 \\
\hline MPADs & Colorimetry & $100-1000$ & 37 & Serum & 44 \\
\hline TMB-Pt@Ag NFs/uricase & Colorimetry & $0.5-150$ & 0.3 & Serum & This work \\
\hline
\end{tabular}

$\mu \mathrm{L}$ of Pt@Ag NFs, but after $30 \mu \mathrm{L}$, it decreased. Therefore, $30 \mu \mathrm{L}$ was chosen as the optimal volume of Pt@Ag NFs. Fig. 3e shows that the relative activity increases with the increase of TMB concentration until saturation. It is worth noting that when the concentration of TMB is more than $1.6 \mathrm{mM}$, the reaction begins to recrystallize due to its low solubility in the aqueous phase. Therefore, the optimal concentration of TMB was $1.6 \mathrm{mM}$ in the following experiments.

\section{Linearity and detection limit}

In the analysis and detection, the sensitivity of the biological enzyme assay is one of the most important factors. In order to verify the sensitivity of Pt@Ag NFs assay for detecting UA, a series of concentrations of UA standard solutions were detected under optimal conditions. With the increase of UA concentration $(0.5-150 \mu \mathrm{M})$, the absorbance of the solution at $652 \mathrm{~nm}$ also increases (Fig. 4a). Moreover, as shown in Fig. 4b, the color of the solution in the centrifuge tube from left to right also showed a visible change (colorless to blue). The illustration of Fig. 4a is a calibration curve for UA concentration and absorbance. There is a linear relationship between $\Delta A$ and UA concentration $(0.5-150 \mu \mathrm{M})$ and the limit of detection (LOD) was $0.3 \mu \mathrm{M}(\mathrm{S} / \mathrm{N}=3)$. The corresponding linear equation is expressed as: $\Delta A=0.0051 C+0.0453(\mu \mathrm{M})$ and the correlation coefficient $\left(R^{2}\right)$ is 0.995 . For comparison, Table 1 summarized the analytical parameters of some systems used to detect uric acid. As can be seen from the table, the method used herein has higher sensitivity and lower detection limit.

\section{Test of selectivity}

To verify the selectivity of the assay in detecting uric acid, we tested several potential interfering substances in human serum, including cysteine, ascorbic acid, glucose, and several inorganic ions. Under the optimum conditions, the following experiments have been carried out. One is the signal of only UA and interfering substances mixed the detection system (Fig. 5 black column). Compared with the absorbance of adding UA, the interference is basically negligible, which proves the specific catalysis of UA by uricase and the assay has a higher selectivity. The other is the response of uric acid, uricase and various interfering substances coexist (Fig. 5 red column). These results indicate that the assay has a special response to UA regardless of the presence or absence of interfering substances.

\section{Real sample detection}

In order to study the feasibility of UA assay, we used the proposed method and biochemistry analyzer to test the serum samples of 5 gout patients. The serum sample was centrifuged at $4000 \mathrm{rpm}$ for $3 \mathrm{~min}$. Then, $5.6 \mu \mathrm{L}$ serum mixed with uricase, 4-aminoantipyrine (4-AAP), and 3,5-diphenylamine disodium salt (MADB). And the samples were put in the biochemical analyzer and measure at $660 / 800 \mathrm{~nm}$. The measurement values of the two methods are almost identical (Table 2), and the average relative error is $3.2 \%$, which means that the developed assay has good accuracy and can be used for the determination of UA in human serum samples. As shown in Table 2, the recoveries of this method are in the range of $96.8-103.3 \%$, and the relative standard deviation (RSD, $n=3$ ) of 5 samples is less than $2.6 \%$. These results show that colorimetric assay for the detection of UA has great practical value in clinical application.

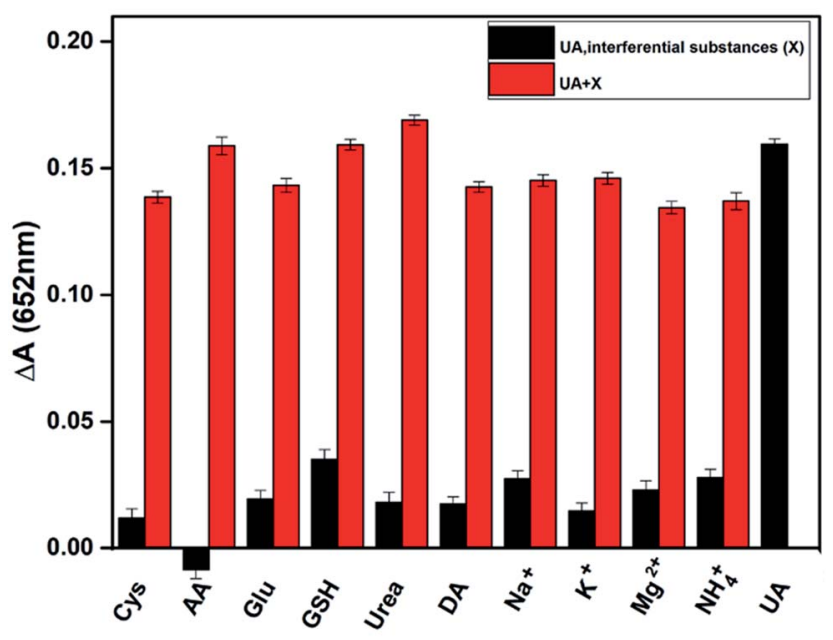

Fig. 5 Selectivity of UA assay. In the absence of UA (black) and presence (red), the interfering substances were added to the reaction solution, respectively. The concentration of UA, ascorbic acid, glutathione and dopamine: $20 \mu \mathrm{M}$, and the concentration of other interfering substances: $200 \mu \mathrm{M}$. 
Table 2 Determination of UA in human serum of goat patients by proposed method and biochemical analysis

\begin{tabular}{|c|c|c|c|c|c|c|c|}
\hline Sample & $\begin{array}{l}\text { Proposed assay } \\
(\mu \mathrm{M})\end{array}$ & $\begin{array}{l}\text { Biochemistry analyzer } \\
(\mu \mathrm{M})\end{array}$ & $\begin{array}{l}\text { Average relative } \\
\text { error }(\%)\end{array}$ & Added $(\mu \mathrm{M})$ & $\begin{array}{l}\text { Found } \\
(\mu \mathrm{M})\end{array}$ & $\begin{array}{l}\text { Recovery } \\
(\%)\end{array}$ & RSD (\%) \\
\hline Serum 1 & 711 & 751 & 3.2 & 500 & 1229 & 103.3 & 1.6 \\
\hline Serum 3 & 936 & 967 & & 500 & 1420 & 96.8 & 2.3 \\
\hline Serum 4 & 522 & 502 & & 500 & 1016 & 98.8 & 1.9 \\
\hline Serum 5 & 482 & 490 & & 500 & 969 & 97.4 & 2.6 \\
\hline
\end{tabular}

\section{Conclusions}

In conclusion, a new assay based on Pt@Ag NFs was constructed to detect UA by the simple colorimetric method. UV-vis spectroscopy showed that Pt@Ag NFs had high sensitivity with the limit of detection for $0.3 \mu \mathrm{M}$ and high selectivity in the detection of UA. The proposed assay was successfully applied to the detection of UA in human serum with the recoveries in the range of $96.8-103.3 \%$, which has the potential of the simple and fast UA detection platform for point-of-care diagnostics.

\section{Compliance with ethical standards}

The study was approved by the ethics committee of Jiangxi Medical College, and their guidelines were followed throughout this study. The serum samples involved in our research were from gout individuals. Informed consent was obtained from all human participants.

\section{Conflicts of interest}

The authors declare that they have no competing interests.

\section{Acknowledgements}

This work was financially supported by the National Natural Science Foundation of China $(21765015,21808099)$ and the Science and Technology Innovation Platform of Jiangxi Province (20192BCD40001), China.

\section{Notes and references}

1 R. Ferin, M. L. Pavao and J. Baptista, Rapid, sensitive and simultaneous determination of ascorbic and uric acids in human plasma by ion-exclusion HPLC-UV, Clin. Biochem., 2013, 46, 665-669.

2 B. N. Ames, R. Cathcart, E. Schwiers and P. Hochstein, Uric acid provides an antioxidant defense in humans against oxidant- and radical-caused aging and cancer: a hypothesis, Proc. Natl. Acad. Sci. U. S. A., 1981, 78, 68586862.

3 W. Chen, Y. Wu, X. Zhao, S. Liu, F. Song, Z. Liu, et al., Screening the anti-gout traditional herbs from TCM using an in vitro method, Chin. Chem. Lett., 2016, 27, 1701-1707.
4 W. L. Nyhan, The recognition of Lesch-Nyhan syndrome as an inborn error of purine metabolism, J. Inherited Metab. Dis., 1997, 20, 171-178.

5 N. Dhaun, J. L. Vachiery, R. L. Benza, R. Naeije, L. J. Hwang, $\mathrm{X}$. Liu, et al., Endothelin antagonism and uric acid levels in pulmonary arterial hypertension: clinical associations, $J$. Heart Lung Transplant., 2014, 33, 521-527.

6 G. Lippi, M. Montagnana, M. Franchini, E. J. Favaloro and G. Targher, The paradoxical relationship between serum uric acid and cardiovascular disease, Clin. Chim. Acta, 2008, 392, 1-7.

7 K. L. Rock, H. Kataoka and J. Lai, Uric acid as a danger signal in gout and its comorbidities, Nat. Rev. Rheumatol., 2013, 9, $13-22$.

8 K. Lin, H. Lin and P. Chou, The interaction between uric acid level and other risk factors on the development of gout among asymptomatic hyperuricemic men in a prospective study, J. Rheumatol., 2000, 27, 1501-1505.

9 V. Pavlicek, P. Tuma, J. Matejckova and E. Samcova, Very fast electrophoretic determination of creatinine and uric acid in human urine using a combination of two capillaries with different internal diameters, Electrophoresis, 2014, 35, 956961.

10 Y. Liu, H. Li, B. Guo, L. Wei, B. Chen and Y. Zhang, Gold nanoclusters as switch-off fluorescent probe for detection of uric acid based on the inner filter effect of hydrogen peroxide-mediated enlargement of gold nanoparticles, Biosens. Bioelectron., 2017, 91, 734-740.

11 A. Fang, Q. Wu, Q. Lu, H. Chen, H. Li, M. Liu, et al., Upconversion ratiometric fluorescence and colorimetric dual-readout assay for uric acid, Biosens. Bioelectron., 2016, 86, 664-670.

12 F. Zhao, Z. Wang, H. Wang, R. Zhao and M. Ding, Determination of uric acid in human urine by ion chromatography with conductivity detector, Chin. Chem. Lett., 2011, 22, 342-345.

13 K. Ngamchuea, C. Batchelor-McAuley and R. G. Compton, Understanding electroanalytical measurements in authentic human saliva leading to the detection of salivary uric acid, Sens. Actuators, B, 2018, 262, 404-410.

14 X. Huang, H. S. Im, O. Yarimaga, J. H. Kim, D. H. Lee, H. S. Kim, et al., Direct electrochemistry of uric acid at chemically assembled carboxylated single-walled carbon nanotubes netlike electrode, J. Phys. Chem. B, 2006, 110, 21850-21856. 
15 T. Khajvand, M. J. Chaichi and A. H. Colagar, Sensitive assay of hexythiazox residue in citrus fruits using gold nanoparticles-catalysed luminol- $\mathrm{H}_{2} \mathrm{O}_{2}$ chemiluminescence, Food Chem., 2015, 173, 514-520.

16 H. Lu, J. Li, M. Zhang, D. Wu and Q. Zhang, A highly selective and sensitive colorimetric uric acid biosensor based on $\mathrm{Cu}$ (II)-catalyzed oxidation of $3,3^{\prime}, 5,5^{\prime}$-tetramethylbenzidine, Sens. Actuators, B, 2017, 244, 77-83.

17 R. K. Bera, A. Anoop and C. R. Raj, Enzyme-free colorimetric assay of serum uric acid, Chem. Commun., 2011, 47, 1149811500.

18 D. Wu, H. Lu, H. Xie, J. Wu, C. Wang and Q. Zhang, Uricasestimulated etching of silver nanoprisms for highly selective and sensitive colorimetric detection of uric acid in human serum, Sens. Actuators, B, 2015, 221, 1433-1440.

19 K. Tan, G. Yang, H. Chen, P. Shen, Y. Huang and Y. Xia, Facet dependent binding and etching: ultra-sensitive colorimetric visualization of blood uric acid by unmodified silver nanoprisms, Biosens. Bioelectron., 2014, 59, 227-232.

20 D. Li, Q. He, Y. Yang, H. Mohwald and J. Li, Two-stage pH response of poly(4-vinylpyridine) grafted gold nanoparticles, Macromolecules, 2008, 41, 7254-7256.

21 J. Lu, Y. Xiong, C. Liao, C. Liao and F. Ye, Colorimetric detection of uric acid in human urine and serum based on peroxidase mimetic activity of MIL-53(Fe), Anal. Methods, 2015, 7, 9894-9899.

22 Q. Lu, J. Deng, Y. Hou, H. Wang, H. Li and Y. Zhang, Onestep electrochemical synthesis of ultrathin graphitic carbon nitride nanosheets and their application to the detection of uric acid, Chem. Commun., 2015, 51, 1225112253.

23 Q. Zhuang, Z. Lin, Y. Jiang, H. Deng, S. He, L. Su, et al., Peroxidase-like activity of nanocrystalline cobalt selenide and its application for uric acid detection, Int. J. Nanomed., 2017, 12, 3295-3302.

24 K. Hirakawa, T. Kaneko and N. Toshima, Kinetics of spontaneous bimetallization between silver and noble metal nanoparticles, Chem.-Asian J., 2018, 13, 1892-1896.

25 P. Biswas, S. Ganguly and P. Dastidar, Stimuli-pesponsive metallogels for synthesizing Ag nanoparticles and sensing hazardous gases, Chem.-Asian J., 2018, 13, 1941-1949.

26 H. You, S. Yang, B. Ding and H. Yang, Synthesis of colloidal metal and metal alloy nanoparticles for electrochemical energy applications, Chem. Soc. Rev., 2013, 42, 2880-2904.

27 A. Wang, Y. Li, M. Wen, G. Yang, J. Feng, J. Yang, et al., Melamine assisted one-pot synthesis of $\mathrm{Au}$ nanoflowers and their catalytic activity towards p-nitrophenol, New J. Chem., 2012, 36, 2286-2291.

28 A. Ruditskiy and Y. Xia, Toward the Synthesis of Sub-15 nm Ag Nanocubes with Sharp Corners and Edges: The Roles of Heterogeneous Nucleation and Surface Capping, J. Am. Chem. Soc., 2016, 138, 3161-3167.

29 C. Morita-Imura, T. Kobayashi, Y. Imura, T. Kawai, H. Shindo, et al., pH-induced recovery and redispersion of shape-controlled gold nanorods for nanocatalysis, RSC Adv., 2015, 5, 75889-75894.
30 J. He, Y. Wang and Y. Feng, Forest of gold nanowires: A new type of nanocrystal growth, ACS Nano, 2013, 7, 2733-2740.

31 E. L. Kunkes, D. A. Simonetti, R. M. West, J. C. Serrano-Ruiz, C. A. Gärtner and J. A. Dumesic, Catalytic conversion of biomass to monofunctional hydrocarbons and targeted liquid-Fuel classes, Science, 2008, 322, 417-421.

32 A. Kowal, M. Li, M. Shao, K. Sasaki, M. B. Vukmirovic, J. Zhang, et al., Ternary $\mathrm{Pt} / \mathrm{Rh} / \mathrm{SnO}_{2}$ electrocatalysts for oxidizing ethanol to $\mathrm{CO}_{2}$, Nat. Mater., 2009, 8, 325-330.

33 E. Formo, E. Lee, D. Campbell and Y. Xia, Functionalization of electrospun $\mathrm{TiO}_{2}$ nanofibers with pt nanoparticles and nanowires for catalytic applications, Nano Lett., 2008, 8, 668-672.

34 L. Kelland, The resurgence of platinum-based cancer chemotherapy, Nat. Rev. Cancer, 2007, 7, 573-584.

35 D. Suh, S. Lee, C. Xu, A. A. Jan and S. Baik, Significantly enhanced phonon mean free path and thermal conductivity by percolation of silver nanoflowers, Phys. Chem. Chem. Phys., 2019, 21, 2453-2462.

36 A. K. Fakhre, C. M. Ajmal and S. Bae, Silver nanoflower decorated graphene oxide sponges for highly sensitive variable stiffness stress sensors, Small, 2018, 14, 18005491800558.

37 S. Tang, H. Shen and Y. Hao, A novel cytosensor based on Pt@Ag nanoflowers and AuNPs/Acetylene black for ultrasensitive and highly specific detection of circulating tumor cells, Biosens. Bioelectron., 2018, 104, 72-78.

38 H. Niu, L. Zhang, J. Feng, Q. Zhang, H. Huang and A. Wang, Graphene-encapsulated cobalt nanoparticles embedded in porous nitrogen-doped graphitic carbon nanosheets as efficient electrocatalysts for oxygen reduction reaction, $J$. Colloid Interface Sci., 2019, 552, 744-751.

39 H. Niu, H. Chen, G. Wen, J. Feng, Q. Zhang and A. Wang, One-pot solvothermal synthesis of three-dimensional hollow $\mathrm{PtCu}$ alloyed dodecahedron nanoframes with excellent electrocatalytic performances for hydrogen evolution and oxygen reduction, J. Colloid Interface Sci., 2019, 539, 525-532.

40 H. Chen, M. Jin, L. Zhang, A. Wang, J. Yuan, Q. Zhang and J. Feng, One-pot aqueous synthesis of two-dimensional porous bimetallic PtPd alloyed nanosheets as highly active and durable electrocatalyst for boosting oxygen reduction and hydrogen evolution, J. Colloid Interface Sci., 2019, 543, 1-8.

41 D. Yao, A. G. Vlessidis and N. P. Evmiridis, Microdialysis sampling and monitoring of uric acid in vivo by a chemiluminescence reaction and an enzyme on immobilized chitosan support membrane, Anal. Chim. Acta, 2003, 478, 23-30.

42 L. Liu, L. Liu, Y. Wang and B. Ye, Novel electrochemical sensor based on bimetallic metal-organic frameworkderived porous carbon for detection of uric acid, Talanta, 2019, 199, 478-484.

43 P. L. D. Santos, V. Katic, K. C. F. Toledo and J. A. Bonacin, Photochemical one-pot synthesis of reduced graphene oxide/Prussian blue nanocomposite for simultaneous 
electrochemical detection of ascorbic acid, dopamine, and uric acid, Sens. Actuators, B, 2018, 255, 2437-2447.

44 E. F. M. Gabriel, P. T. Garcia, T. M. G. Cardoso, F. M. Lopes, F. T. Martins and W. K. T. Coltro, Highly sensitive colorimetric detection of glucose and uric acid in biological fluids using chitosan-modified paper microfluidic devices, Analyst, 2016, 141, 4749-4756.

45 G. Yue, S. Li, W. Liu, F. Ding, P. Zou, X. Wang, et al., Ratiometric fluorescence based on silver clusters and N, Fe doped carbon dots for determination of $\mathrm{H}_{2} \mathrm{O}_{2}$ and UA: N, Fe doped carbon dots as mimetic peroxidase, Sens. Actuators, B, 2019, 287, 408-415.

46 X. Wang, G. Zhu, W. Cao, Z. Liu, C. Pan, W. Hu, et al., A novel ratiometric fluorescent probe for the detection of uric acid in human blood based on $\mathrm{H}_{2} \mathrm{O}_{2}$-mediated fluorescence quenching of gold/silver nanoclusters, Talanta, 2019, 191, 46-53. 